\title{
UNA PROPUESTA DE INTERVENCIÓN INTERCULTURAL PARA LA EDUCACIÓN SECUNDARIA EN CANTABRIA
}

\author{
María Louzao Suárez \\ Lourdes Durántez Pascual \\ I.E.S. Miguel Herrero Pereda (Torrelavega)
}

\begin{abstract}
RESUMEN: Las demandas que la sociedad del siglo XXI hace a la escuela en cuanto a la necesidad de formar a la ciudadanía para una sociedad multicultural, así como el hecho real de la presencia de un colectivo de alumnado cada vez más diverso y multicultural en las aulas está provocando que, desde los propios centros educativos, se pongan en marcha propuestas pedagógicas que, a nuestro entender, deben tener un doble objetivo: facilitar la escolarización del alumnado extranjero y trabajar con el conjunto del alumnado (especialmente el autóctono) en la aceptación de la diversidad, promoviendo su competencia intercultural.

Desde hace un tiempo, en la Comunidad Autónoma de Cantabria, se viene trabajando en esta línea, tratando de afrontar algunas situaciones que observamos en el centro como: la dificultad para el éxito escolar del alumnado extranjero y la influencia que esto tiene en su ajuste personal; la presencia de actitudes interculturales condicionadas por el contexto familiar y social del alumnado, y su resistencia al cambio; la ausencia de contenidos interculturales reales en el currículo; la escasa flexibilidad del sistema educativo para adaptarse a los cambios; o la importancia de una formación permanente como "iluminación" de la práctica, entre otras.
\end{abstract}

PALABRAS CLAVE: Educación secundaria, educación intercultural, plan de interculturalidad.

\section{A PROPOSAL FOR INTERCULTURAL INTERVENTION IN SECONDARY EDUCATION IN CANTABRIA}

\footnotetext{
ABSTRACT: The modern society of the XXI century is creating a great number of demands that have to be covered by our school system referring to the need to educate citizens in a multicultural society and to the increasing amount of
} 
foreign and multicultural students in our classrooms. This is producing a great number of pedagogical activities in our schools which should have a double purpose: to facilitate the enrollment of foreign students and to work with all students (especially native) in the acceptance of diversity and promoting intercultural competence.

For a time, in the Community of Cantabria has been working in this field, trying to address some situations we observe in the centre as: the difficulty for foreign students to achieve academic success and the influence this has on their personal development, the presence of cultural attitudes conditioned by the family and social background of these students and their resistance to change, the absence of a real cross-cultural content in the curriculum, the resistance of the education system to adapt to changes, the importance of lifelong learning as "enlightenment" of practice, etc.

KEYWORDS: Secondary education, intercultural education, intercultural plan.

Recibido: 15/01/2011

Aceptado: 14/10/2011

\section{INTRODUCCIÓN: NUESTRO PUNTO DE PARTIDA}

Los cambios sociales, políticos, económicos, geográficos, etc., acontecidos a consecuencia de la globalización han favorecido la apertura de fronteras y la movilización de grupos humanos en busca de mejores condiciones de vida. La presencia en espacios físicos de realidades culturales diferentes es ahora mucho más evidente, especialmente en países y regiones históricamente "emigrantes" y poco acostumbradas a la recepción de personas de otros lugares.

Ante esta nueva situación, parece que nuestro lugar como profesorado del siglo XXI es aceptar la multiculturalidad como una de las características fundamentales de la sociedad actual. Dado que la labor de la escuela es formar ciudadanía, se entiende que es un aspecto destacable de nuestro compromiso profesional promover la toma de conciencia de esta realidad, tanto en la comunidad educativa como a la hora de ayudar a la escuela a que ésta contribuya a construir en la ciudadanía dicha toma de conciencia. Pues, como es evidente, que esta situación sea así no tiene relación directa y causal con que todos los grupos humanos estén de acuerdo en que hay que hacer algo específico en esa formación de la ciudadanía para una sociedad multicultural (baste con ver el repunte de los partidos políticos con ideologías racistas y xenófobas en varios países europeos durante los últimos años).

Se entiende, pues, que nuestra realidad concreta puede ser más o menos multicultural, pero la sociedad, en general, sí lo es y por eso nuestro proyecto docente en la educación pública va encaminado a promover la competencia intercultural en el conjunto de la comunidad educativa, entendiendo que aquélla no se puede reducir de ningún modo a la presencia de alumnado extranjero en el centro y que se ha de promover, con independencia de situaciones concretas y/o de "proyectos políticos determinados". 
La situación actual de los centros no es, pues, fácil. La educación secundaria se perfila, a nuestro entender, como una etapa de especial complejidad por muchos motivos: desde la propia organización escolar, con una rigidez estructural histórica y una reticencia a los cambios y a las innovaciones, con una tradición disciplinar fuertemente arraigada que prioriza contenidos alejados de problemáticas sociales y relevantes para el alumnado; una práctica educativa poco flexible para valorar cómo adquirir conocimientos, habilidades y estrategias propias de otras tradiciones curriculares y culturales; e, incluso, la falta de formación del profesorado, que se encuentra con situaciones en el aula que desbordan su capacidad para darles una respuesta.

A estos obstáculos internos debe añadirse las dificultades específicas que tiene el alumnado extranjero de incorporación tardía al sistema educativo: la complejidad de las materias en cuanto a su dificultad para el aprendizaje y la interferencia del aprendizaje lingüístico cuando se desconoce la lengua del proceso de aprendizaje; el desconocimiento de las rutinas escolares y de las pautas culturales propias del nuevo contexto educativo; las propias experiencias escolares previas; la situación afectiva y emocional del alumnado, en su doble condición de adolescente y extranjero, en función de su situación familiar y de su estatus socioeconómico, así como la falta de comprensión de su nueva realidad, que generalmente él no ha elegido. No se esconde, sin embargo, que la observación y el tratamiento de algunos de los problemas enunciados ha permitido orientar la acción educativa para encontrar un sitio en la escuela a este colectivo de alumnos y alumnas, comprobando que algunas de las medidas incidían positivamente en el conjunto del alumnado y mejoraban la escuela.

El centro escolar sigue siendo un cauce fundamental para la integración tanto del alumnado extranjero como de su familia. Se comparte con Besalú (2010) el hecho de que aquél sigue siendo un espacio privilegiado, por su propia naturaleza, para incorporar la dimensión intercultural. Se acuerda con Besalú y Vila (2007), y Besalú (2008) en que la educación intercultural no es tan reciente como puede parecer. El sistema educativo es consciente de las necesidades que esta nueva realidad le demanda y también de la oportunidad que esto representa (Torres, 2008), aspecto que se quiere aprovechar para ofrecer a nuestra comunidad educativa un plan de centro, en el marco del modelo educativo y un Plan de Interculturalidad de la comunidad autónoma de Cantabria, que promueva prácticas educativas que, dentro de sus posibilidades, puedan contribuir a construir una escuela con un proyecto de futuro para toda la ciudadanía, presidido por la igualdad y la cohesión social y el logro de los derechos humanos para todos (Banks, 2005; Besalú, 2008; Grant, 2009).

Las investigaciones que se vienen realizando durante los últimos años (Louzao y González, 2007; Louzao, 2009) habían puesto de manifiesto que los esfuerzos de los centros educativos se dirigían fundamentalmente a la puesta en marcha de medidas y estrategias con un carácter eminentemente compensador de las dificultades que presentaba el alumnado extranjero (retraso curricular y/o desconocimiento de la lengua), siendo muy escasa la didáctica de la interculturalidad, la incorporación de un planteamiento didáctico centrado en contenidos sociales.

El plan de trabajo que presentamos, a continuación, fruto de nuestra experiencia en el IES Miguel Herrero Pereda de Torrelavega (Cantabria, España), pretende ofrecer un alcance más global, superando esa visión restrictiva de entender la interculturalidad como la atención educativa al alumnado inmigrante (Besalú, 2008) e, 
incluso, trascendiendo la idea de multiculturalidad y sus significados (Grant, Sleeter y Anderson, 1988). Ante la realidad que se nos plantea en este centro (y seguramente en muchos otros), se entiende que la educación intercultural supone un enfoque holístico inclusivo que, partiendo del respeto y la valoración de la diversidad cultural, busca la reforma de la escuela como totalidad, para incrementar la equidad educativa, superar el racismo/discriminación/exclusión, favorecer la comunicación y la competencia interculturales y apoyar el cambio social según principios de justicia social (Gil Jaurena, 2002).

\section{Nuestra PROPUESTA de TRABAJO: El PLAN DE ACCIÓN O PLAN DE INTERCULTURALIDAD DE CENTRO}

El plan de intervención realizado en el IES Miguel Herrero de Torrelavega nace en el curso 2006-2007, siguiendo las directrices del Plan de Interculturalidad de la Consejería de Educación de Cantabria, con la intención de dar respuesta a la diversidad cultural que empezaba a estar presente en el mismo. Dado que las experiencias previas de escolarización de alumnado inmigrante eran prácticamente inexistentes, la comunidad educativa se empieza a encontrar repentinamente ante una nueva situación muy novedosa para la cual no estaba preparada.

La mayor parte del alumnado autóctono tampoco había tenido experiencias escolares previas de contacto con alumnos y alumnas de otros países lo que, unido a los cambios que en determinados barrios de la ciudad se estaban produciendo simultáneamente, hizo necesaria la intervención educativa, dirigida tanto a tratar de resolver las necesidades del alumnado que se incorporaba como a promover actitudes interculturales positivas y desarrollar la competencia intercultural con todo el alumnado.

De acuerdo al modelo educativo de Cantabria, y ante la realidad detectada, la administración educativa promueve el establecimiento en los centros educativos de todas las etapas de la figura del coordinador/a de interculturalidad (un profesor/a del claustro), persona encargada de facilitar la integración de alumnado extranjero y movilizar la educación intercultural en el centro. Además, el Plan de Interculturalidad pone en marcha otros recursos externos para facilitar la tarea en los centros: las aulas de dinamización intercultural (ADI), el asesor de interculturalidad en los Centros de Profesores (CEP) y los auxiliares de lengua de origen (ALO). Partiendo de esta estructura, el coordinador/a, en colaboración con el equipo de interculturalidad, desarrolla y pone en marcha un Plan de Centro, cuyas líneas generales, en nuestro caso, se comentan a continuación.

\section{El primer pilar: los recursos humanos. El coordinador/a de interculturalidad / equipo de interculturalidad del centro}

El coordinador/a de interculturalidad (un profesor/a del claustro sensibilizado e interesado en la cuestión) es la pieza clave de todo este entramado. Sin embargo, necesita de un conjunto de compañeros y compañeras que, constituyendo el equipo de interculturalidad del centro, le apoyen en su labor. 
1a. El coordinador/a de interculturalidad. Su función principal es observar el centro y anticipar una propuesta de actuación (el Plan de Acción) que oriente las actuaciones a realizar. Se trata de estudiar qué alumnado inmigrante tenemos escolarizados en el centro, qué tipo de atención precisa y cómo se va a prestar ésta. En segundo lugar, establecer qué actuaciones y recursos se establecen entre los equipos docentes. En tercer lugar, hacer las propuestas de educación intercultural para trabajar con la comunidad educativa.

Es tarea de la coordinación sensibilizar a los Equipos Directivos y al Claustro planteando la educación intercultural como una propuesta educativa para el conjunto del alumnado del centro y como oportunidad para la renovación curricular y metodológica y, por ello, debe quedar reflejado en los documentos organizativos del mismo. Dicho Plan de Acción estaría formado por dos grandes proyectos de trabajo: el Plan de Atención Docente y el Plan de Intervención Intercultural.

$2^{\text {a }}$. El Equipo de Interculturalidad. Se cree que la tarea no puede ser de una sola persona en un centro. En nuestro caso, se promueve la constitución de un Equipo, constituido por:

a) Un conjunto de profesores y profesoras (6/8), con perfiles diferentes -saben lenguas, son de diferentes departamentos, tienen experiencia y están sensibilizados-, además del orientador/a y un Jefe/a de Estudios que, con número variable de horas, dediquen una parte de su trabajo escolar a desarrollar el Plan de Centro. Sus funciones son las siguientes: imparten español como lengua extranjera (L2), apoyan en las áreas, ayudan en el asesoramiento a los departamentos didácticos, asisten a las evaluaciones, acompañan el proceso de aprendizaje del alumnado y valoran su progreso, y colaboran decisivamente en las actuaciones interculturales. El Equipo, además, refuerza la presencia de la educación intercultural como aspecto educativo, crea una estructura organizativa nueva y actúa como grupo de presión, no solo ante las resistencias que presentan determinados sectores de la comunidad educativa ante la llegada de inmigrantes sino, también, ante la inercia y la falta de comprensión racional de la nueva realidad. La tarea del Coordinador/a se convierte, entonces, en tarea de un Equipo.

b) Departamento de Orientación/Jefatura de Estudios. La colaboración que ofrece el Departamento de Orientación es tan amplia como se pueda requerir: asesoramiento al equipo de interculturalidad para la ubicación del alumnado extranjero en los grupos; el seguimiento individualizado a través del desarrollo de la acción tutorial; el apoyo en el trabajo a los departamentos didácticos a la hora de adaptar medidas de atención a la diversidad con el alumnado que lo precise; orientar la respuesta académica y/o profesional; la inclusión en el Plan de Acción Tutorial y en el marco de las programaciones didácticas de las actividades y propuestas para trabajar la competencia intercultural con todo el alumnado; la dinamización de la formación continua del profesorado y, por ende, la promoción de la renovación curricular y pedagógica; incluso, finalmente, en el trabajo directo con el alumnado que precisa el español como L2. La jefatura de 
estudios se encargaría de ofrecer los espacios y tiempos para el desarrollo de todas estas actividades.

c) Es imprescindible destacar también la labor del Equipo Directivo, ya que sin éste no hay plan de centro. Es preciso que proporcione las horas, los espacios, los recursos y la flexibilidad organizativa necesaria para dar respuesta a las necesidades de estos alumnos. Es necesaria una relación de confianza y colaboración estrecha entre un equipo y otro y, sin duda, es una oportunidad para flexibilizar, adaptar e imaginar un espacio escolar donde, y en la medida de lo posible, la organización esté al servicio de la educación; donde las rutinas, sin duda necesarias, no se impongan a las nuevas necesidades. Hemos comprobado cómo la presencia de alumnado extranjero ha forzado a encontrar "otras" soluciones organizativas, que lentamente va creando una estructura escolar más capaz de dar respuesta a las nuevas realidades.

d) Auxiliar de Lengua de Origen (ALO), personas que tienen como lengua materna alguna de las lenguas de origen de nuestro alumnado y que realizan una labor fundamental de colaboración con todo el equipo de interculturalidad y el profesorado mismo. Este perfil profesional ha sido una propuesta incorporada en el Plan de Interculturalidad de Cantabria, aprovechando otras figuras profesionales ya existentes en el marco de otros programas europeos como Comenius. Su presencia en el equipo ha permitido encontrar nuevas líneas de trabajo en la adaptación lingüística de los materiales de aula, en la intervención directa en el proceso de aprendizaje del alumnado al proporcionar las claves en lengua materna para la comprensión del conocimiento escolar. Su intervención ha sido muy eficaz en la comunicación con el alumnado y sus familias, en el trabajo y asesoramiento a los equipos docentes y en la colaboración directa con la coordinadora y/o con la orientadora del centro en el asesoramiento académico y profesional del alumnado. Igualmente, ha sido un apoyo muy importante en el resto de actividades interculturales que se han puesto en marcha en el centro.

El equipo cuenta, además, con el apoyo y el asesoramiento externo de las Aulas de Dinamización Intercultural (ADI), cuya función principal es apoyar y colaborar con los centros y con la colaboración en tareas de formación del CEP (Centro del Profesorado).

\section{El segundo pilar: el Plan de acción}

\section{El plan de atención docente}

El Plan de Interculturalidad para nuestro centro se organiza en torno a dos ejes fundamentales: el Plan de Atención Docente (dirigido principalmente a trabajar con el profesorado en la propuesta educativa del alumnado extranjero) y las Propuestas de Intervención Intercultural a nivel de centro (propuesta educativa orientada a promover la competencia intercultural en el conjunto de la comunidad educativa).

Comenzando por el primero de los ejes, se considera relevante destacar que el Plan de Atención Docente es la hoja de ruta que recoge el conjunto de actuaciones edu- 
cativas necesarias para compensar la desigual situación en la que se encuentra el alumnado extranjero, respecto del autóctono, para poder tener éxito escolar. Se organiza en torno a tres ejes básicos: aprendizaje del español (L2), aprendizaje de las áreas (lengua de instrucción) y, por último, apoyo y seguimiento de las tareas escolares. Consta, todo ello, de actuaciones a cuatro niveles: actuaciones con el alumnado inmigrante, con sus familias, con los equipos educativos y con el conjunto del alumnado.

$1^{\circ}$. Actuaciones con el alumnado inmigrante, que abarcan desde la fase inicial de acogida en el centro y en el aula, la atención lingüística en el caso de desconocer la lengua de aprendizaje de la escuela, el apoyo a la inmersión en el aula y el seguimiento académico a lo largo de toda la escolarización en el centro. Su implicación también es núcleo fundamental en las actividades de centro que tratan de promover la educación intercultural.

$2^{\circ}$. Actuaciones con las familias inmigrantes, a través de un protocolo de actuación orientado a facilitar la comunicación: informar, escuchar y solicitar su colaboración en el proceso educativo y en la evolución académica de sus hijos e hijas; igualmente, se pretende favorecer su acercamiento al centro y su implicación en el conjunto de actividades interculturales que se llevan a cabo con el conjunto de la comunidad educativa.

$3^{\circ}$. Actuaciones con el profesorado, orientadas a la sensibilización, información, asesoramiento, coordinación y formación. El equipo de interculturalidad tiene un primer papel relevante al presentar a los alumnos a los equipos docentes. La coordinación y las propuestas de intervención educativa, las orientaciones metodológicas y didácticas (adaptaciones lingüísticas y de materiales, organización del trabajo de aula, diversificación del currículo, aprendizaje lingüístico) y asesoramiento sobre materiales y recursos, constituye una parte fundamental del trabajo en equipo que debe, por tanto, estar en proceso de formación. Además, se considera aspecto fundamental estimular y orientar la formación en el propio centro (grupos de trabajo, seminarios) que elabore propuestas de acción y reflexión, promueva pequeños cambios y experiencias de innovación y renovación educativa que son necesarias para adaptarse y dar respuesta a las nuevas demandas que la sociedad hace a la escuela pública.

$4^{\circ}$. Actuaciones con el conjunto del alumnado. Se apuntaba previamente, en la introducción de este trabajo, que uno de los objetivos de nuestro Plan de Interculturalidad era aprovechar la diversidad existente y ampliar la intervención intercultural más allá de las medidas para paliar las necesidades educativas del alumnado de origen extranjero. Una verdadera educación intercultural necesita de cambios en todos los elementos del currículo que incidan directamente sobre el proceso de enseñanza-aprendizaje. Sin embargo, dada la complejidad de los cambios que es preciso llevar a cabo, se entiende que, al menos, pueden aprovecharse los espacios y los tiempos para trabajar la interculturalidad con el conjunto del alumnado, siendo uno de ellos la hora semanal de tutoría. No obstante, las actividades que se Ilevan a cabo en el centro para difundir las lenguas y las culturas de origen diferentes a las autóctonas, permiten hacer ciertas "incidencias" en las programaciones didácticas, dando así un mayor alcance a las realizadas en el marco de la acción tutorial. 


\section{El plan de intervención intercultural}

El objetivo fundamental que se plantea es introducir la perspectiva intercultural en el conjunto de planes y proyectos educativos que hay en el centro, ofreciendo una visión de conjunto a toda la comunidad educativa: Proyecto Educativo y Curricular y todo lo que ellos incorporan (plan de acción tutorial, plan de atención a la diversidad, plan de convivencia, programaciones didácticas, etc.). Se desea promover el desarrollo de una sólida competencia cultural que capacite a todo el alumnado para funcionar en sociedades multiculturales y multilingües. El Plan de Acción Tutorial sigue siendo un marco concreto en el que se pueden desarrollar actividades interculturales con todo el alumnado, pero procurando extender la incorporación a las programaciones didácticas de la perspectiva de los problemas sociales relevantes como contenidos centrales a trabajar con el alumnado.

Además, se propone hacer visibles lenguas y culturas existentes en el centro (Coelho, 2006). Con carácter anual se propone el acercamiento al país de origen del alumnado del centro. Se trabaja en conjunto con los departamentos didácticos, quienes proponen actividades en relación con su programación de aula, con las familias inmigrantes, con colaboradores y entidades culturales externas al centro. Se ha revelado como una forma de trabajo interesante para implicar a los departamentos en un proyecto anual común: refuerza los vínculos afectivos y emocionales del alumnado extranjero y sus familias, así como sugiere la idea de otras formas y fuentes de acceso al conocimiento. A lo largo de estos últimos cursos nuestro alumnado ha podido acercarse al conocimiento de las culturas rumano-moldavas, árabe y, en un futuro próximo, a la china (1).

Finalmente, otro aspecto relevante del Plan de Intervención Intercultural es la formación docente: se entiende que el objetivo de favorecer la renovación metodológica y curricular implica crear una escuela con más "conciencia de la realidad" y emprender procesos de formación continua a nivel de centro puede verse favorecido por el intercambio de necesidades, preocupaciones, ideas y experiencias que entendemos no ofrecen directamente "recetas" para resolver la práctica, pero que sí pueden ser útiles para "iluminarla". En el momento actual se considera que una propuesta de formación interesante es la constitución de un Grupo de Trabajo a nivel de centro, que reúne al profesorado interesado en formarse y reflexionar sobre la propia práctica, poniendo el foco de atención en este caso, entre otras cuestiones relacionadas con la interculturalidad, en la adaptación de los materiales curriculares para su posterior utilización en el aula.

\section{Nuestras conclusiones y aportaciones}

Dado que lo que aquí se presenta no es un proyecto de investigación en sentido estricto, pero sí el resultado de un proceso de puesta en marcha y evaluación de nuestro trabajo, se ofrecen algunas reflexiones que, más que resultados, seguramente constituyen nuestras preocupaciones presentes y futuras. Se quiere señalar, sin embargo, que la presencia dentro del centro del equipo de interculturalidad ha significado avances significativos, a la vez que pone en evidencia que la mayor dificultad sea promover la perspectiva intercultural como mirada educativa en el 
centro. Éstos son, a nuestro juicio, algunos de los avances logrados a partir de la existencia del plan de acción intercultural en el centro:

- La presencia del componente afectivo-emocional en el proceso educativo. Las acciones y actuaciones programadas refuerzan el vínculo afectivo del alumnado extranjero con la escuela (condición imprescindible para que se pueda producir el aprendizaje), minimiza los errores derivados del desconocimiento cultural y presenta al nuevo alumnado como portador de valores, conocimientos y experiencias que enriquecen a todos. De esta forma se transmiten valores transversales capaces de ilustrar "otras maneras" de enfocar las relaciones humanas y las diferencias culturales.

El componente actitudinal juega aquí, a nuestro entender, un importante papel (Louzao y González, 2009). Por un lado, las actitudes del alumnado autóctono, son un componente esencial para la integración social del alumnado que se incorpora. Dependiendo del contexto socio-cultural en el que se ubique el centro y, por tanto, atendiendo al perfil de nuestro alumnado autóctono y al de sus familias, la valoración que los grupos hacen de la interculturalidad es diferente. Si el sentido de la misma es negativo, se observa que las ideas previas que tienen los chicos y las chicas resultan resistentes al cambio y están ya bastante afianzadas, lo cual dificulta la acogida del alumnado extranjero que se incorpora al centro. El resultado, la dificultad añadida a la académica que tiene éste para integrarse socialmente en dicho espacio. De hecho, en nuestro caso, es frecuente observar que, con independencia de la nacionalidad, el alumnado extranjero ocupa un espacio concreto en las horas de recreo, sin participar en muchos casos de actividades colectivas con otros compañeros españoles. Por otro lado, las propias actitudes del alumnado extranjero, que son también decisivas en todo el proceso de aprendizaje y de integración. Con independencia de su variedad, hay que tener en cuenta varios aspectos que lo afectan: aspectos académicos ya conocidos como su escolarización previa, expectativas familiares hacia el estudio y la escuela, similitud de la lengua materna con la nueva lengua a adquirir, similitudes entre las pautas culturales previas (tanto académicas como sociales) y las actuales, entre otras cuestiones; pero también aspectos personales que tienen que ver con el proyecto migratorio: decisión, en muchos casos unilateral, por parte de las familias de emprender el viaje, la vivencia de un proceso de reunificación familiar después de varios años sin convivencia con los padres, arraigo al lugar de origen, etc. Así, es habitual que al llegar al centro pasen por una fase inicial de desconcierto, amplificada por las dificultades escolares y lingüísticas con las que se encuentran. En algunos casos, transcurrido cierto tiempo, se observa también que el arraigo se va reconstruyendo en relación a la sociedad de acogida, disminuyendo el apego por la sociedad de origen, rechazando incluso el contacto con compañeros nuevos de su misma nacionalidad.

- Introduce procesos de innovación y cambios. Lo que se viene observando es que la mayor parte de los cambios realizados en el aula, supuestamente para atender al alumnado con desconocimiento del español, benefician también al 
resto. Los esfuerzos hechos por una parte del profesorado para enseñar la lengua de instrucción, la confección de materiales adaptados lingüísticamente, las propuestas de nuevas situaciones de aprendizaje en el aula o el uso de recursos diversos (imágenes, traductores, la red), utilizados para facilitar el aprendizaje del alumnado extranjero, terminan modificando la práctica de aula en beneficio de una mayoría de alumnas y alumnos nativos cuyo dominio del español tampoco resulta suficiente para adquirir el conocimiento escolar. No escondemos, con esto, que el mayor reto planteado con los primeros es la enseñanza-adquisición de la competencia lingüística académica que les permita no sólo acceder a los contenidos de las distintas áreas sino, también, aprender destrezas, estrategias y procedimientos de estudio transferibles a cualquier área de conocimiento.

Sin embargo, siguen observándose las limitaciones de la propia organización escolar para ofrecer una igualdad de oportunidades y la adopción de una verdadera mirada intercultural en los centros. Ni los contenidos seleccionados, ni la organización de los espacios y los tiempos, ni la formación inicial y permanente del profesorado, ni los recursos humanos y materiales existentes, ofrecen garantías suficientes para que aquellos colectivos que se incorporan en situación de desventaja (sea ésta de la naturaleza que sea) y que se encuentran en mayor riesgo de sufrir exclusión social, puedan promocionar con las mismas oportunidades que los demás. Como bien han comprobado Huguet y Navarro (2006), el fracaso escolar del alumnado inmigrante es considerablemente mayor que el del alumnado autóctono, y hay más posibilidades de sufrir fracaso cuanto más tardíamente se incorporan a nuestro sistema educativo. En este sentido, el trabajo diario permite observar que los centros escolares no tienen todavía la capacidad necesaria para adaptarse a las incorporaciones progresivas de alumnos y alumnas, sobre todo si éstas se producen una vez comenzado el curso escolar y esta limitación es tanto más evidente cuanto más altos sean los niveles de enseñanza en los que se escolarizan.

Profundizando en ello, y en lo que respecta al profesorado, consideramos que hay dos cuestiones que dificultan la educación intercultural en los centros: en primer lugar, su escasa formación en estos temas; dicha formación sigue siendo una pieza clave para favorecer una educación intercultural (Soriano, 2009; Jordán, 1996), entendiendo por ésta una nueva oportunidad de promover cambios en los centros y mejoras en las prácticas profesionales. Aquélla no puede quedar reducida a la labor del coordinador/a de interculturalidad ni al equipo de interculturalidad de los centros. Las administraciones educativas han de incentivar dicha formación con propuestas que motiven al profesorado a invertir tiempo y esfuerzos. La autoformación en los centros, en colaboración con los Centros de Profesores y con otros colegas que estén desarrollando labores similares a las nuestras, se nos antoja como un elemento imprescindible de mejora de nuestra práctica profesional. El intercambio de experiencias, compartir materiales y la evaluación en su puesta en práctica, así como la lectura de documentos que fomentan la reflexión y animan al cambio, constituyen uno de los puntos fuertes de nuestro Plan de Interculturalidad. 
En segundo lugar, la permanencia en el centro durante un corto período de tiempo de una parte del profesorado involucrado en el proyecto, que cambia de destino en las convocatorias oficiales de movilidad docente, limita la necesaria continuidad que sería preciso dar para que las propuestas mencionadas anteriormente se fueran asentando en el currículo y la organización escolar. La puesta en marcha de procesos formativos en el propio centro, no obstante, permite contextualizar la ayuda y "movilizar" a los compañeros y compañeras que han de enfrentarse por vez primera a la presencia de la diversidad cultural y lingüística en su aula. El hecho de encontrarse en el aula con alumnado que tiene diferentes niveles de competencia en la lengua de instrucción es ahora mismo una de las principales preocupaciones del profesorado (Huguet, Navarro, Chireac y Sansó, 2009) y así lo constatamos en nuestro centro. Los tiempos escolares y las exigencias del día a día lo impacientan y esto repercute en el desarrollo personal del alumnado extranjero, pues se observa que las dificultades que tiene una parte del profesorado para trabajar la competencia lingüística académica en el aula provoca situaciones pasivas de aprendizaje y una dependencia excesiva del equipo de interculturalidad, tanto en el plano personal como académico por parte, además, no sólo de esta parte del profesorado, sino también del propio alumnado extranjero (McLaughlin, 2006).

En lo que respecta a los espacios y los tiempos, destacaríamos las dificultades encontradas para el desarrollo de un buen trabajo en equipo, provocadas en parte por los escasos espacios y tiempos con los que contamos para las labores de coordinación y colaboración. El coordinador/a impulsa, pero no puede abarcar por sí mismo/a todos los aspectos del plan de interculturalidad. La riqueza de este equipo es precisamente su multidisciplinariedad y su coordinación. El apoyo del equipo directivo y de la jefatura de estudios es otro pilar fundamental. Además, la reciente incorporación de las ALO (auxiliar de lengua de origen) supone una ayuda decisiva para el equipo, pues su labor de mediación con el alumnado, con las familias y con el profesorado permite solventar algunas de las dificultades mencionadas.

- Induce a nuevas formas de organización y trabajo escolar. La presencia en el centro del equipo de interculturalidad (al margen de cualquier órgano escolar), las formas de trabajo (cambios y flexibilidad horaria a lo largo del curso), la presencia de apoyos dentro de las aulas (ALOS y profesorado de apoyo), la propuesta de actividades conjuntas a los departamentos didácticos, la creación de materiales y recursos para dar respuesta a las exigencias del currículo, el uso de diferentes lenguas en las tareas escolares, así como la colaboración de familias e instituciones en actividades escolares, son algunas de las pequeñas innovaciones que se han introducido en el centro. Precisamente la implicación de las familias inmigrantes es, como la de las familias autóctonas, bastante escasa, aunque sí enormemente positiva cuando colaboran en el centro en las actividades interculturales que se proponen como medio para acercar a todo el alumnado al conocimiento de otras culturas. Tanto las ALO como las Aulas de Dinamización Intercultural ofrecen el recurso de la mediación familiar, especialmente en lo que se refiere a labores de traducción y comunicación, facili- 
tando enormemente el intercambio y la toma de decisiones conjuntas en aquellos aspectos que afectan a los alumnos.

Para concluir y, ante todas estas consideraciones, se hace necesario volver a llamar la atención sobre el hecho de que son necesarios cambios globales y profundos en la institución escolar. Las propias políticas educativas habrían de dejar de ser propuestas abstractas para convertirse en reales pero, para ello, es evidente que las circunstancias actuales habrían de cambiar. Ello debería ir unido igualmente a otro tipo de políticas sociales que contribuyeran a ofrecer una mayor equidad social.

\section{NOTAS}

El conjunto del trabajo que se viene desarrollando en el centro a lo largo de los últimos cursos se puede consultar en la página web del mismo: www.iesmiguelhe rrero.com.

\section{REFERENCIAS BIBLIOGRÁFICAS}

BANKS, J. (2005). Cultural diversity and education: foundations, curriculum and teaching. Boston: Pearson Education.

BESALÚ, X. (coord.) (2007). Educar en sociedades pluriculturales. Barcelona: Wolters Kluwer.

BESALÚ, X. (2010). La educación intercultural y el currículo escolar. Primer Congreso Internacional en la red sobre interculturalidad y educación. Extraído el 20 de julio de 2010, de http://www.aulaintercultural.org/IMG/pdf/besalu-2.pdf.

BESALÚ, X. y VILA, I. (2007). La buena educación. Libertad e igualdad en la escuela del Siglo XX. Madrid: Catarata.

COELHO, E. (2006). Enseñar y aprender en escuelas multiculturales. Barcelona: Horsori.

CUADRADO, I. y MUÑOZ, M. (2008). Respuestas organizativas a la interculturalidad. Presentación. Organización y Gestión Educativa, 4, 14.

GIL JAURENA, I. (2002). La educación intercultural en la enseñanza obligatoria: una guía para su evaluación y desarrollo. Madrid: UNED. [trabajo de investigación inédito].

GRANT, C. (2009). Una voz en pro de los derechos humanos y la justicia social: la educación intercultural como herramienta para promover las promesas y evitar los riesgos de la globalización. En E. Soriano (Coord.), Vivir entre culturas: una nueva sociedad (pp. 25-51). Madrid: La Muralla.

GRANT, C.; SLEETER, C. y ANDERSON, J. E. (1988). The literature of multicultural education: Review and analysis. Part II. Educational Studies, 13, 47-71.

HUGUET, A. y NAVARRO, J. (2006). Inmigración y escuela. Revista interuniversitaria de formación del profesorado, 56, 17-22.

HUGUET, Á.; NAVARRO, J. L.; CHIREAC, S-M. y SANSÓ, C. (2009). Edad de llegada y lengua de la escuela. Consideraciones a partir de un estudio con alumnado inmigrante. Revista de Psicología General y Aplicada, 62 (3), 219-229. 
JORDÁN, J. A. (1996). Propuestas de educación intercultural para profesores. Barcelona: CEAC.

LOUZAO, M. (2009). Diseño, desarrollo y evaluación de un proyecto de educación intercultural en un centro asturiano de Educación Primaria. Una perspectiva de la investigación en la acción y el estudio de caso. Tesis doctoral inédita. Oviedo: Universidad de Oviedo.

LOUZAO, M. y GONZÁLEZ, J. A. (2007). La integración social y educativa del alumnado inmigrante en Asturias. Un estudio de caso. En Los Premios Nacionales de Investigación Educativa y Tesis Doctorales 2005 (pp. 11-41). Madrid: CIDE.

MCLAUGHLIN, B. (2006). Mitos y falsas realidades acerca de una segunda lengua: lo que todo profesor debería olvidar. Textos de Didáctica de la Lengua y de la Literatura, 41, 71-83.

SORIANO, E. (2009). La formación del profesorado para construir una nueva sociedad. En E. Soriano (Coord.), Vivir entre culturas: una nueva sociedad (pp. 53-83). Madrid: La Muralla.

TORRES, X. (2008). Diversidad cultural y contenidos escolares. Revista de Educación, 345, 83-110. 